Research Article

\title{
Offline Single-Polarization Radar Quantitative Precipitation Estimation Based on a Spatiotemporal Deep Fusion Model
}

\author{
Yonghong Zhang $\mathbb{D}^{1}{ }^{1}$ Shiwei Chen, ${ }^{1}$ Wei Tian, ${ }^{2}$ Guangyi Ma, ${ }^{3}$ and Shuai Chen ${ }^{1}$ \\ ${ }^{1}$ School of Automation, Nanjing University of Information Science \& Technology, Nanjing, China \\ ${ }^{2}$ School of Computer \& Software, Nanjing University of Information Science \& Technology, Nanjing, China \\ ${ }^{3}$ School of Electronic \& Information Engineering, Nanjing University of Information Science \& Technology, Nanjing, China
}

Correspondence should be addressed to Yonghong Zhang; zyh@nuist.edu.cn

Received 6 April 2021; Revised 8 June 2021; Accepted 18 July 2021; Published 28 July 2021

Academic Editor: Federico Porcù

Copyright ( 2021 Yonghong Zhang et al. This is an open access article distributed under the Creative Commons Attribution License, which permits unrestricted use, distribution, and reproduction in any medium, provided the original work is properly cited.

\begin{abstract}
Quantitative precipitation estimation (QPE) based on Doppler radar plays an important role in severe weather monitoring, industrial and agricultural production, and natural disaster prediction and prevention. However, the temporal and spatial variability of precipitation leads to large errors in radar estimates of mixed precipitation. To improve the accuracy of radar QPE, we propose an offline spatiotemporal deep fusion model that uses the reflectivity data of the Shijiazhuang Doppler radar Z9311 and the precipitation data from 17 national weather stations (NWSs) and 260 automatic weather stations (AWSs). Considering the abrupt spatial changes in precipitation, a three-dimensional radar data structure is proposed, and the spatial features of multielevation and multiscale radar data are extracted and merged using the feature fusion network (FFNet). Finally, the time dependence of the precipitation is captured using the long short-term memory (LSTM) network, and the precipitation estimation is obtained. Based on a comparison of the results of the proposed model (FFNet-LSTM) with those of the ordinary kriging (OK) interpolation, two Z-R relationship, the multilayer perceptron (MLP), the LSTM, and the FFNet, the proposed method is superior to these models, has a promising performance, and is a general-purpose rainfall algorithm.
\end{abstract}

\section{Introduction}

A more accurate and real-time quantitative precipitation estimation (QPE) provides a reliable data source for hydrology, meteorology, and disaster forecasting $[1,2]$. However, the spatial distribution of the rainfall process is extremely complicated, and $70-80 \%$ of the uncertainty of the terrestrial hydrological process is attributed to the temporal and spatial variability of precipitation, which poses a severe challenge in QPE [3,4]. A rain gauge is a tool commonly used in QPE, which can provide accurate measurements at point locations. However, the sparse network of rain gauges leads to a low spatial coverage [5]. The spatial resolution of radar data is the best of all types of remote sensing data [6]. While the spatial resolution of satellite images is greater than $5-10 \mathrm{~km}$, the spatial resolution of radar data is about $1 \mathrm{~km}$ [7-9]. Therefore, radar data are often used in QPE research due to their better temporal and spatial resolution [10-12], but precipitation estimations using radar data contain various errors. The large-scale deployment of dual-polarization weather radar has not yet been achieved in mainland China [13], and it is difficult to obtain its data. Therefore, this research is based on single-polarization radar data. Hereinafter, single-polarization radar is referred to as radar for convenience. Currently, the QPE methods based on rain gauge and radar data include two types - the rain gauge interpolation method and the radar conversion method.

In previous studies, the Kriging algorithm is the most widely used and studied rain gauge interpolation methods [14]. The Kriging interpolation method obtains the precipitation value at an unknown location by estimating the continuous attribute value between the rain gauges. The methods based on Kriging all make the basic assumption of Gaussian characteristics, and the fitting of the variogram 
model is complicated $[15,16]$. In order to relax the Gaussian assumption and make the interpolation method more flexible, some researchers have conducted experiments from different angles. Lu and Wong [17] studied the spatial structure of data that cannot be effectively modeled using a typical variogram and proposed an adaptive inverse distance weighting (AIDW) spatial interpolation technique. Chen et al. [18] compared five interpolation methods based on daily precipitation data from 753 stations in mainland China; these were the ordinary nearest neighbor, local polynomial, radial basis function, inverse distance weighting (IDW), and ordinary kriging (OK) methods. They concluded that the OK and IDW methods rank the highest in terms of the quality of the interpolation of the daily precipitation in China. To obtain the variogram of the OK method with better adaptability in daily precipitation interpolation, Wang et al. [19] compared and analyzed the four commonly used variograms (Exponential, Spherical, Gaussian, and Linear). The experimental results show that the Exponential and Spherical functions have good adaptability to the OK method in daily precipitation interpolation. However, rain gauges have several drawbacks, such as poor spatial representation, limited spatial coverage, and uneven spatial distribution $[20,21]$. Therefore, the performances of precipitation estimations based on the rain gauge interpolation method are unsatisfactory in practice.

The $Z-R$ relationship is a traditional radar conversion QPE algorithm. The rainfall intensity is calculated using the nonlinear relationship between the radar reflectivity $(Z)$ and the precipitation rate $(R)$ [22-24]. In early research, the simple $Z-R$ relationship was obtained statistically in different climate zones, and then a quantitative estimation of the precipitation was realized $[25,26]$. Ryde predicts various weather systems based on radar reflectivity of different sizes $(5-10 \mathrm{~cm})$ [27]. Based on China's New Generation Weather Radar (CINRAD) system data, the QPE in mainland China follows the fixed relationship of $Z=300 R^{1.4}$ [28]. Limited by the technical conditions at the time, most radar reflectivity and rain gauge measurement data contain errors, and the $Z$ $R$ relationship cannot be corrected in time. Moreover, the parameters in the $Z-R$ relationship are affected by factors such as synoptic weather situations, hydrology, and geography, thus varying in time and space [29-33]. Such a situation makes the $Z-R$ relationship an idealized model that has difficulty reproducing the actual conditions.

Many studies aimed to reduce radar QPE errors based on the Z-R relationship. Brandes and Edward [34] used the ratio between the radar estimates and the rain gauge measurement data as a correction factor (without considering the spatial variation between the ratios) to optimize the $Z-R$ relationship. Yuan et al. [35] proposed an improved least squares method as a discriminant function to optimize the parameters in the fixed $Z-R$ relationship. They summarized the $Z-R$ relationship optimized by the optimization method, and both the average relative error at the station and the relative error of area rainfall are greatly reduced. Ramli et al. [36] divided the rainfall into seven categories according to the rainfall intensity and season and derived new $Z-R$ relationships using the optimization method, which significantly improved the radar
QPE accuracy. They also concluded that the $Z-R$ relationship depends largely on the location and type of rainfall. Similar conclusions have been obtained in other studies; that is, the $Z$ $R$ relationship does not apply to all regions [37-39].

Radar QPE research based on data-driven methods has been conducted for many years, such as artificial neural networks (ANNs) [40, 41], nonparametric methods [42], support vector machines (SVMs) [43], and random forest (RF) [44]. Unlike the $Z-R$ relationship, these methods attempt to directly capture the relationship between the radar reflectivity and the rain gauge measurements. Kusiak et al. [41] used a multilayer perceptron (MLP) to estimate the rainfall intensity on multiple time scales, and the estimation results were more accurate than those obtained using the four data-mining algorithms (i.e., the RF, classification and regression tree, SVM, and K-nearest neighbor). Tang et al. [5] explored the time dependence of precipitation and constructed a continuous conditional random field (CCRF) with geographic and time weighting for radar QPE, which produced a more accurate precipitation estimate than the $Z$ $R$ relationship. Liu et al. [45] studied the local spatial correlation of rainfall using a radial basis function neural network (RBFNN) with local mapping characteristics to build an adaptive neural network (ANN), which considered the variability of the relationship between the radar reflectivity and the rainfall estimation. Liu et al. [46] demonstrated that reflectivity $1-4 \mathrm{~km}$ above the rain gauge is the best input vector for the RBFNN. The spatial variation during the rainfall process was fully considered by Xiao and Chandrasekar [47] and Trafalis et al. [48]. They conducted a preliminary study on the structure of the three-dimensional radar data; that is, they obtained constant altitude plan position indicating (CAPPI) data at different altitudes above a ground station. Three-dimensional radar data can reflect the spatial structure of the rainfall and be used to obtain more information that may affect the precipitation estimate. However, the above research uses the same size radar data at different altitudes, regardless of the influence of the horizontal advection. The data observed by the radar are related to the rainfall rate on the ground, with a certain time delay and spatial movement [22]. Therefore, three-dimensional radar data that use CAPPI of the same size at different altitudes contain more information that has nothing to do with precipitation. Chiang et al. [49] considered the influence of the horizontal advection in the atmosphere and concluded that the coverage of 3D radar data at each altitude is related to the horizontal wind speed and the vertical (terminal) velocity of the raindrops. Therefore, before designing the size of the data grid, it is necessary to determine the time it takes the raindrops to reach the ground from different altitudes. They assumed a raindrop size of $4 \mathrm{~mm}$, calculated the terminal velocity using an empirical formula, and obtained the time for the raindrop to reach the ground from the CAPPI at different altitudes. The horizontal offset was determined by assuming the tangential wind speed in the area. Finally, they determined the size of the CAPPI at different altitudes and established a radar QPE and a quantitative precipitation prediction (QPF) in Taiwan based on a dynamic ANN. 
Although the above methods considered the time dependence and spatial correlation of the precipitation to a certain extent and have achieved good results, the model in the previous study is only suitable for point-shaped radar data due to the structural characteristics. The input of the model is multiple data points in three-dimensional radar data, which cannot describe the real rainfall process. In addition, the estimated precipitation time scale is usually 1 hour or longer in these studies. Compared with the models used in these studies, the precipitation estimates on the 6minute time scale are intense and susceptible to noise, especially the estimation of light rain $[50,51]$. In this study, a radar QPE method based on the spatiotemporal deep fusion model is proposed to solve the abovementioned problems. It is expected to further improve the accuracy of radar QPEs because the new method considers the spatial variations and temporal integrity of the precipitation simultaneously. The rest of this manuscript is structured as follows. Section 2 presents the information about the study area and the data source. Section 3 describes the details of the model and the evaluation metrics. Section 4 covers the evaluation of the proposed model and the comparison models at a 6-minute time scale. The main conclusions of the study are summarized in Section 5.

1.1. Study Area and Data Source. The study area is over Shijiazhuang $\left(37^{\circ} 27^{\prime}-38^{\circ} 47^{\prime} \mathrm{N}, 113^{\circ} 30^{\prime}-115^{\circ} 20^{\prime} \mathrm{E}\right.$ ) (red area in the inset in Figure 1) and spans two major geomorphological units: the Taihang Mountains and the North China Plain. The study area is low in the southeast and high in the northwest, with a large elevation difference and complex topography. Under the influence of the topographic uplift, the precipitation in the region decreases from west to east, and the rainfall is concentrated in the summer (JuneSeptember) [41]. The typical characteristics of the summer precipitation in the study area are a high precipitation intensity, short duration, and strong locality. The heavy rain in the area is difficult to predict and is often underreported [52-54]. Therefore, the local short-term mixed precipitation estimation is the focus of our research.

All the data used in this study were obtained from the Shijiazhuang Meteorological Center (SMC), including January to December 2017-2019 (UTC+8) Z9311 Doppler radar reflectivity and National Weather Stations (NWSs) data and Automatic Weather Stations (AWSs) data from January to December 2018 (UTC+8). The precipitation data from the NWSs are part of a national ground weather station precipitation dataset established by the China National Meteorological Center based on long-term historical data and quality control. $97.5 \%$ of NWS data have an accuracy rate higher than 99\%, which have reliable authenticity [55]. The data quality of the AWSs is affected by factors such as the observation instrument, observation technique, station location, observation time, and observation method. Various nonclimatic factors have a greater impact on the ground meteorological data obtained via long-term observations [56]. The rainfall resolution of the AWSs is $0.1 \mathrm{~mm}$ per minute, and the maximum error is $\pm 3 \%$. In this study, the Grubbs algorithm $[57,58]$ was used to control the quality of the AWSs precipitation data. Specifically, the abnormal values in the precipitation data within 3 hours were removed, which improved the authenticity of the AWSs precipitation data. Affected by the East Asian monsoon, the water vapor transported by the East Asian monsoon in June begins to affect the study area. The monsoon enters an active period from July to August, providing a large amount of water vapor for precipitation in the area. In late August, the subtropical high retreats south, but the water vapor carried by the monsoon can still affect the area during this retreat [59]. Therefore, the precipitation data from June to September each year were used as the research data. As shown in Table 1, the Doppler radar Z9311 is located in Xinle, Shijiazhuang $\left(38^{\circ} 21^{\prime} 6^{\prime \prime} \mathrm{N}, 114^{\circ} 42^{\prime} 42^{\prime \prime} \mathrm{E}\right)$, and the effective scanning radial distance is $230 \mathrm{~km}$. The radar completes 9 volume scans with different elevation angles in 6 minutes $\left(0.49^{\circ}, 1.40^{\circ}, 2.38^{\circ}, 3.20^{\circ}, 4.27^{\circ}, 5.93^{\circ}, 9.79^{\circ}, 14.50^{\circ}\right.$, and $\left.19.42^{\circ}\right)$, and the scan mode is the volume coverage pattern, scan strategy \#2, version 1 (VCP21). Therefore, the radar file is updated every 6 minutes. To better obtain the spatial structure of the precipitation, the CAPPI was used as the radar data in the research. Specifically, reflectivity of less than $0 \mathrm{~dB}$ was eliminated, and then the original polar coordinate radar data were converted into reflectivity in Cartesian coordinates, with a grid size of $1 \times 1 \mathrm{~km}$. In addition, the missing data at a certain elevation were interpolated using a Cressman weighting function (Equations (1) and (2)) [60]. The radius used in the $X, Y$, and $Z$ directions was $1.5 \mathrm{~km}, 1.5 \mathrm{~km}$, and $1 \mathrm{~km}$, respectively. Finally, the CAPPIs of different elevations were generated with each rain gauge as the center.

$$
\begin{array}{r}
R^{2}=\mathrm{d} X^{2}+\mathrm{d} Y^{2}+\mathrm{d} Z^{2}, \\
W(r)= \begin{cases}\frac{R^{2}-r^{2}}{R^{2}+r^{2}}, & r \leq R, \\
0, & r>R .\end{cases}
\end{array}
$$

In equations (1) and (2), $R$ is the radius of influence and and $r$ is the distance between the gate and the grid point.

Correspondingly, there are 17 NWSs and 260 AWSs within the coverage of this radar (Figure 1). The data from NWSs and AWSs were calculated every 1 minute. To match the precipitation and reflectivity data, we marked the reflectivity according to time. The radar reflectivity data recording time was taken as a mark, the corresponding precipitation data were located, and the rain gauge rainfall in the previous 6 minutes was collected. This is assumed that the recording time of the radar data file is $T$ and the time of the collected precipitation data file is $T, T-1, T-2, \ldots, T-5$. The unit of the generated precipitation data is $\mathrm{mm} / 6 \mathrm{~min}$. Then, based on the longitude and latitude of the weather station, we matched the reflectivity with the precipitation. The precipitation and reflectivity data were also labeled based on time. 


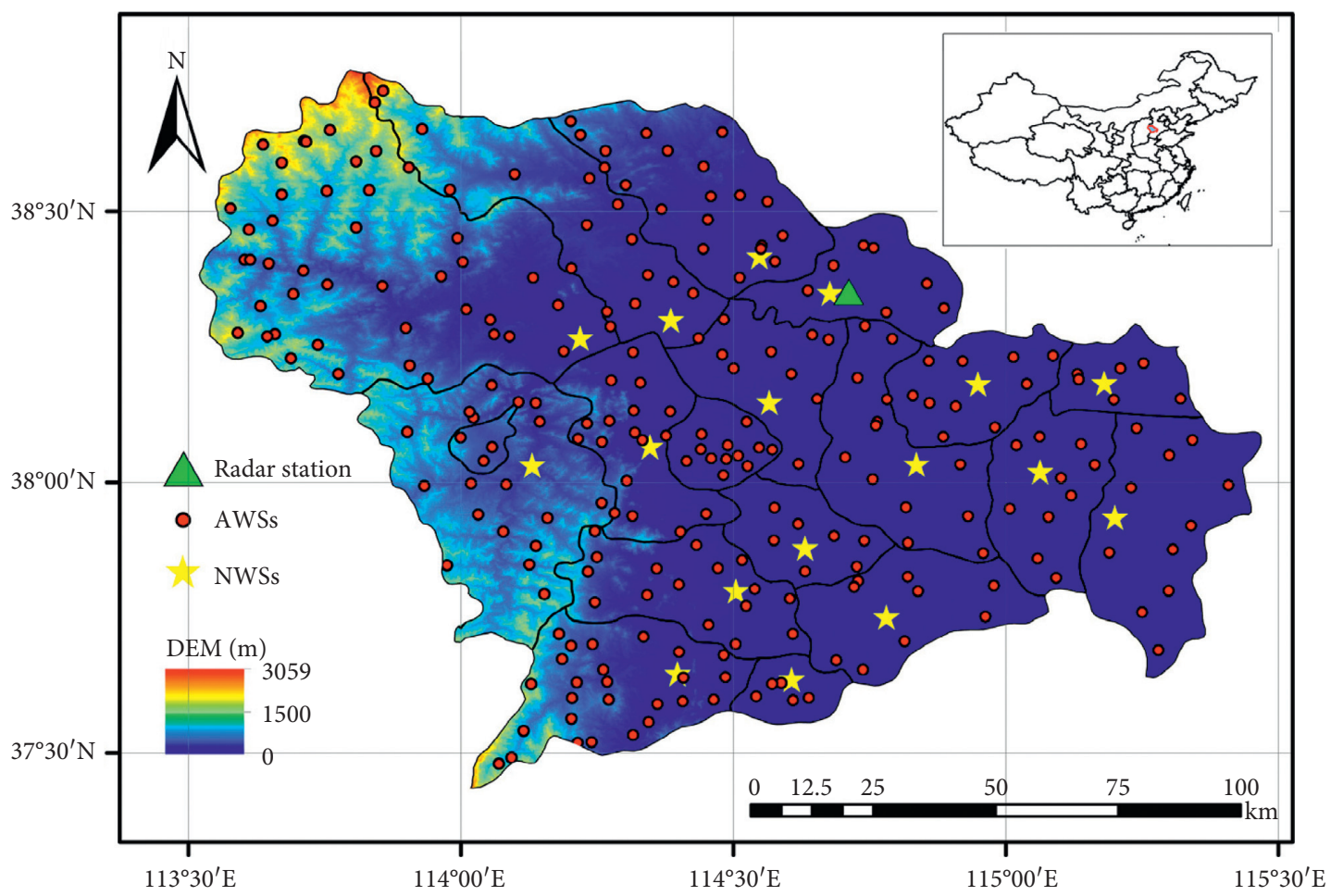

FIgURE 1: Location of the study area, rain gauges, and radar stations.

TABLE 1: Technical characteristics of Doppler radar Z9311.

\begin{tabular}{lc}
\hline Characteristic & Value \\
\hline Position & $114^{\circ} 42^{\prime} 42^{\prime \prime} \mathrm{E} 38^{\circ} 21^{\prime} 6^{\prime \prime} \mathrm{N}$ \\
Height (above ground level) & $19.42 \mathrm{~m}$ \\
Polarization type & Single-polarization \\
Wavelength & $10 \mathrm{~cm}(S$-band) \\
Maximum range & $460 \mathrm{~km}$ \\
Useful range & $230 \mathrm{~km}$ \\
Bin resolution & $1 \mathrm{~km}$ \\
Scan interval (VCP21) & $6 \mathrm{~min}$ \\
No. of elevations (VCP21) & $9\left(0.49^{\circ}-19.42^{\circ}\right)$ \\
\hline
\end{tabular}

\section{Methodology}

The proposed deep learning-based spatiotemporal deep fusion model framework is shown in Figure 2. The entire process can be divided into three parts: (1) data preprocessing, including geometric correction, time alignment, conversion of raw radar data into CAPPI, training, verification, and test data acquisition; (2) combining the FFNet and LSTM to establish a spatiotemporal deep neural network model; and (3) evaluation of the precipitation estimation model, which includes training, verifying, and testing the proposed model based on the data and quantifying the performance of the model through certain evaluation indicators.

2.1. Data Processing. Thorndahl et al. [38] proposed the three-dimensional radar data structure shown in Figure 3. The grid size of the $0.5 \mathrm{~km}$ elevation is $3 \times 3 \mathrm{~km}^{2}$, the grid size of the $1.0 \mathrm{~km}$ elevation is $7 \times 7 \mathrm{~km}^{2}$, the grid size of the
$1.5 \mathrm{~km}$ elevation is $11 \times 11 \mathrm{~km}^{2}$, and the grid size of the $2.0 \mathrm{~km}$ elevation is $15 \times 15 \mathrm{~km}^{2}$. In addition, they concluded that the correlation between the reflectivity of the lowest elevation and the rainfall is more significant and stable than at the other three elevations, and the variability at the three higher elevations is greater than that at the lowest elevation. Therefore, the 9 data points at the lowest height and the average reflectivity of the three higher elevations were taken as the input, for a total of 12 data points. However, this method greatly destroys the spatial correlation between the three-dimensional data structure, and it is also difficult to capture the relationship between the high-level reflectivity and the ground rain gauge. According to Hao et al. [61], the rainfall in the study area from June to September in the past years did not exceed $150 \mathrm{~mm}$ per month and the average rainfall intensity of the heavy rain over the years was $75 \mathrm{~mm} / \mathrm{d}$. A method of sorting and averaging based on two parameters (SATP) [62] is used to establish the relationship between the rainfall intensity $R$ and the raindrop volume diameter $D$, and it is concluded that the raindrop size of the study area does not exceed $4 \mathrm{~mm}$, which is the same size as the data assumed in the study of Thorndahl et al. [38]. Therefore, the same three-dimensional radar data structure was also used in our research. The difference is that instead of the average reflectivity at the three higher altitudes, we used all the grid values at the higher altitudes as the input and merged the reflectivity at the different altitudes.

The best training data period of the radar precipitation model is within 1 hour [45]; that is, the precipitation within 1 hour has a strong correlation. Therefore, based on the continuity of the precipitation process, we used 10 


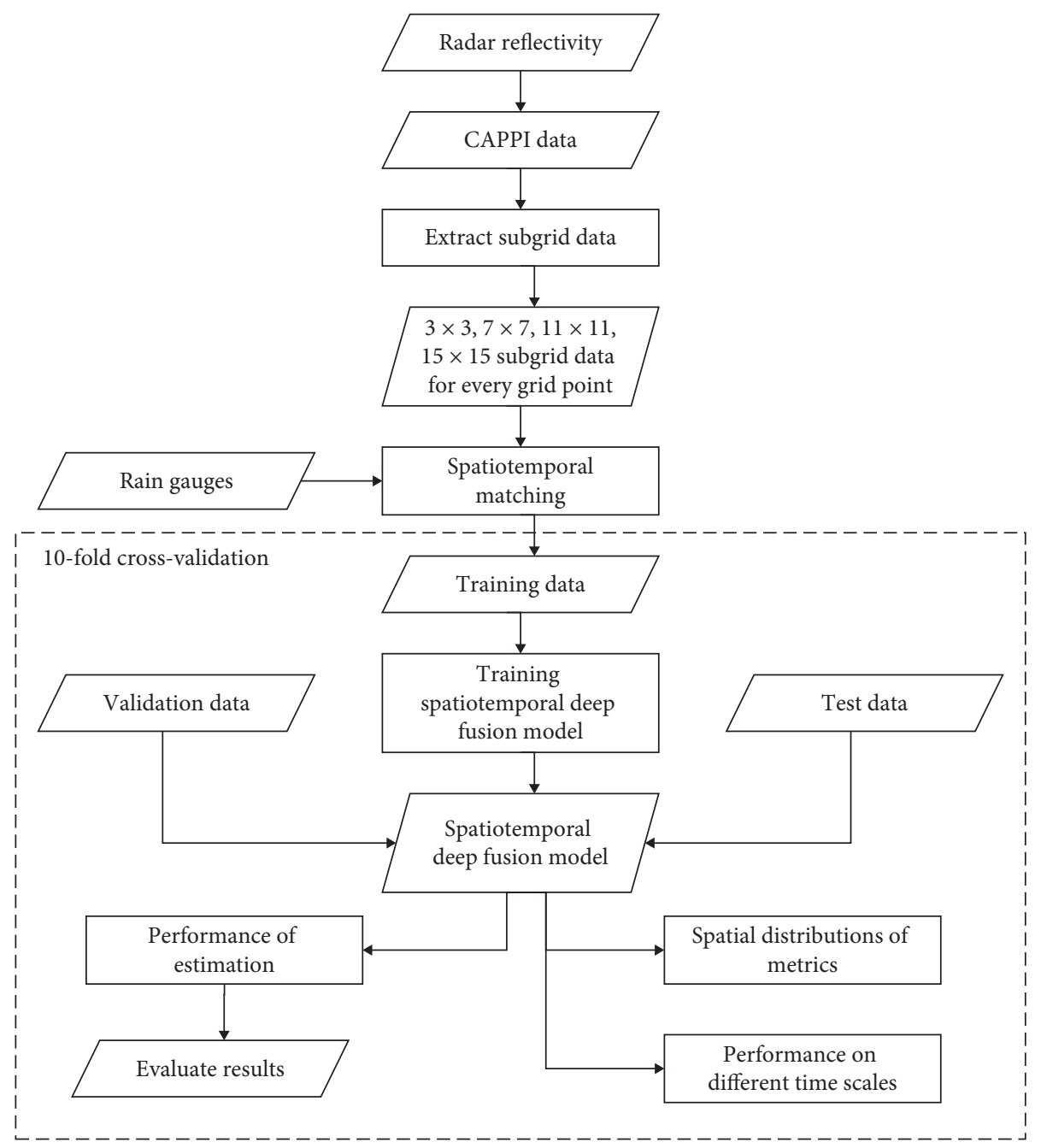

FIgURE 2: Flow chart of the spatiotemporal deep integration network.

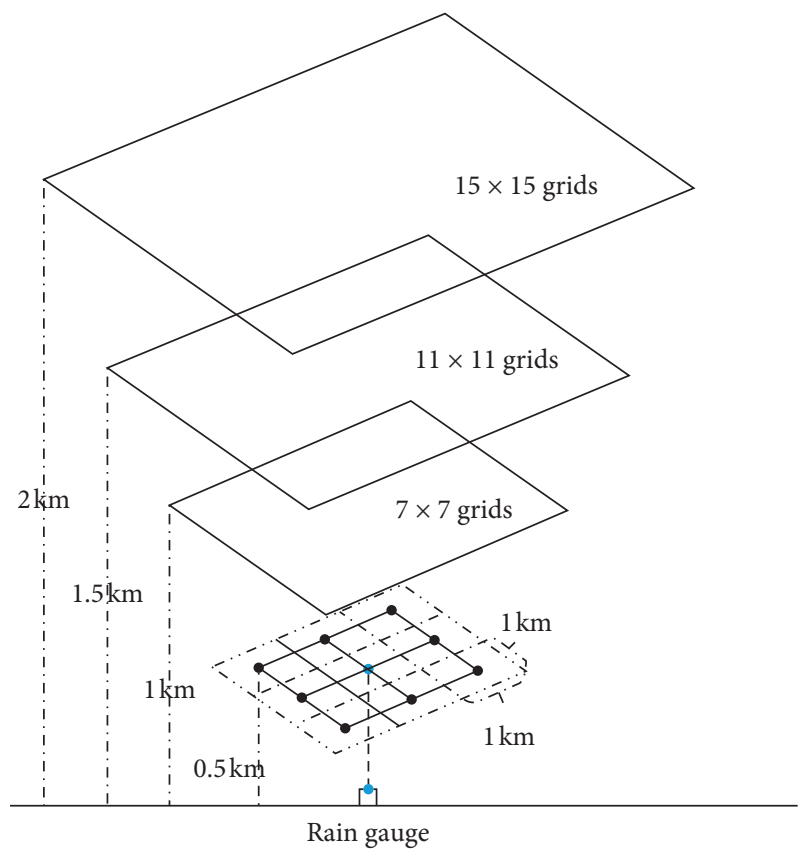

Figure 3: The three-dimensional radar data structure. precipitation data and 10 three-dimensional radar data within 1 hour as a precipitation sample of a station to estimate the precipitation at the last timestamp within an hour. Finally, 16,603 and 43,324 precipitation samples were generated based on the NWSs and AWSs data, respectively.

2.2. Basic Neural Network Models. Feature fusion networks (FFNets) are widely used in the field of computer vision, such as image segmentation [63], target tracking [64], and content-aware image resizing [65]. An FFNet mainly uses convolutional neural networks (CNNs) to extract target features in a layer-by-layer abstract manner. A CNN can automatically extract the important feature information from the image through the view of the convolution kernel and the combined characteristics of the neurons. Therefore, it can capture the spatial features of two-dimensional data. In addition, an FFNet fuses two feature maps using the addition algorithm, so it can extract the edge information better. In the estimation of the precipitation, the spatial distribution of the rainfall above the rain gauge is complicated, and it is difficult to accurately estimate the rainfall on the ground rain gauge through the radar reflectivity 
information for a certain elevation. The rainfall information for different elevations can be integrated through the FFNet to establish the relationship between the high-level radar features and the ground rain gauge. Moreover, the precipitation information for each grid point is related to the surrounding information, and the spatial dependence of the target grid and the surrounding area is automatically learned through the different perception domains in the CNN.

A recurrent neural network (RNN) is a neural network commonly used to process sequence data. Specifically, the RNN layer has feedback connections, which allow the past information to affect the current input. Through this structure, the RNN can remember past information. Long short-term memory (LSTM) is a special RNN, which can solve the problem of gradient disappearance and gradient explosion in the training process of long sequence data. For longer sequences, the LSTM performs better than the ordinary RNN. The structure is shown in Figure 4.

The LSTM is composed of three internal gates: the input gate $i$, the output gate $o$, and the forget gate $f$. These structures control the information of the input and output memory units, optimize the hidden state $h_{t}$, and obtain $C_{t}$ using the following equations:

Gate vectors:

$$
\begin{aligned}
i_{t} & =\sigma\left(W_{i} \cdot\left[h_{t-1}, x_{t}\right]+b_{i}\right), \\
o_{t} & =\sigma\left(W_{o}\left[h_{t-1}, x_{t}\right]+b_{o}\right), \\
f_{t} & =\sigma\left(W_{f} \cdot\left[h_{t-1}, x_{t}\right]+b_{f}\right) .
\end{aligned}
$$

Adapt:

$$
\widetilde{C}_{t}=\tanh \left(W_{C} \cdot\left[h_{t-1}, x_{t}\right]+b_{c}\right) .
$$

Update status:

$$
\begin{aligned}
C_{t} & =f_{t} * C_{t-1}+i_{t} * \widetilde{C}_{t}, \\
h_{t} & =o_{t} * \tanh \left(C_{t}\right) .
\end{aligned}
$$

In equations (3)-(8), $W$ and $b$ are the matrix weights and deviations, respectively; $i_{t}, o_{t}$, and $f_{t}$ are the gate vectors; $\sigma$ is the sigmoid function; $x_{t}$ is the input vector; and $*$ denotes element-wise multiplication. Specifically, the united state $C_{t}$ is used to store the accumulation of past information, and the forget gate $f_{t}$ determines how much of the cell state at the previous moment is retained to the current time, and the input gate $i_{t}$ determines how much information of the network input is saved to the cell state at the current time, and the output gate $o_{t}$ determines how much information the cell state outputs. Precipitation is a continuous process, and the precipitation information for each timestamp is related to the precipitation information in the past time. Therefore, the time dependence of the precipitation information at different timestamps can be obtained using the LSTM.
2.3. Spatiotemporal Deep Fusion Model (FFNet-LSTM). In order to capture the spatial and temporal correlations between the rain gauge and radar reflectivity simultaneously, an FFNet was used to blend the spatial features of the multielevation and multisize radar reflectivity, and the LSTM was used to capture the time dependence. Finally, a spatiotemporal deep fusion network combining the FFNet and LSTM was developed to achieve radar quantitative precipitation estimation. Figure 5 illustrates the framework of the proposed model.

The spatiotemporal deep fusion network includes two parts: the FFNet model and the LSTM model. In the precipitation process, the precipitation at each spatial location does not exist in isolation but is associated with the surrounding space. The FFNet extracts the feature vectors related to the precipitation at the grid center points from the multielevation and multisize 3D radar reflectivity data and merges them from high level to low level. The FFNet is divided into a feature extraction function and a feature fusion function, which is composed of multiple convolutional layers with different convolution kernel sizes and a matrix addition algorithm. The four network features are flattened and concatenated as the output of the model. Given the integrity constraints of the time series, the FFNet network is built on the LSTM network and the complete sequence is returned. It is difficult for a single-layer LSTM network to learn complex spatiotemporal characteristics in a shallow structure, and the use of multilayer LSTM units gives the model the ability to learn by leaps and bounds. The ground rainfall is affected by the weather conditions before and after the timeline. A bidirectional-LSTM (Bi-LSTM) can not only learn past information but can also capture future information. Therefore, based on the continuity of precipitation, a multilayer BiLSTM model was used to obtain the time dependence of the precipitation. The output of the fusion network at the last time stamp $\left(R_{10}\right)$ was used as the value of the radar QPE.

The precipitation samples from the NWSs were randomly divided into 10 parts using ten-fold cross-validation, nine of which were used for training and the remaining one was used for validation at each time (Figure 6). The precipitation samples from the AWSs were used as the test set. The training dataset was used to optimize the model parameters and prevent overfitting. To build a regression model using deep learning algorithms, the hyperparameters of the model should be adjusted during the training phase. The initial values of the parameters were randomly selected from the standard normal distribution. The mean square error (MSE) was defined as the minimum loss function to calculate the error between the estimated value and the true value (Equation (9)). The output of the FFNET-LSTM network is multi-time stamp precipitation, which contains the output of the time stamp considered and for the 9 previous time stamps; and the estimated precipitation of the time stamp considered is the output of the final model, so we propose two forms of loss function: Loss_ $T_{10}$ (Equation (10)) and Loss_Sum (Equation (11)). 


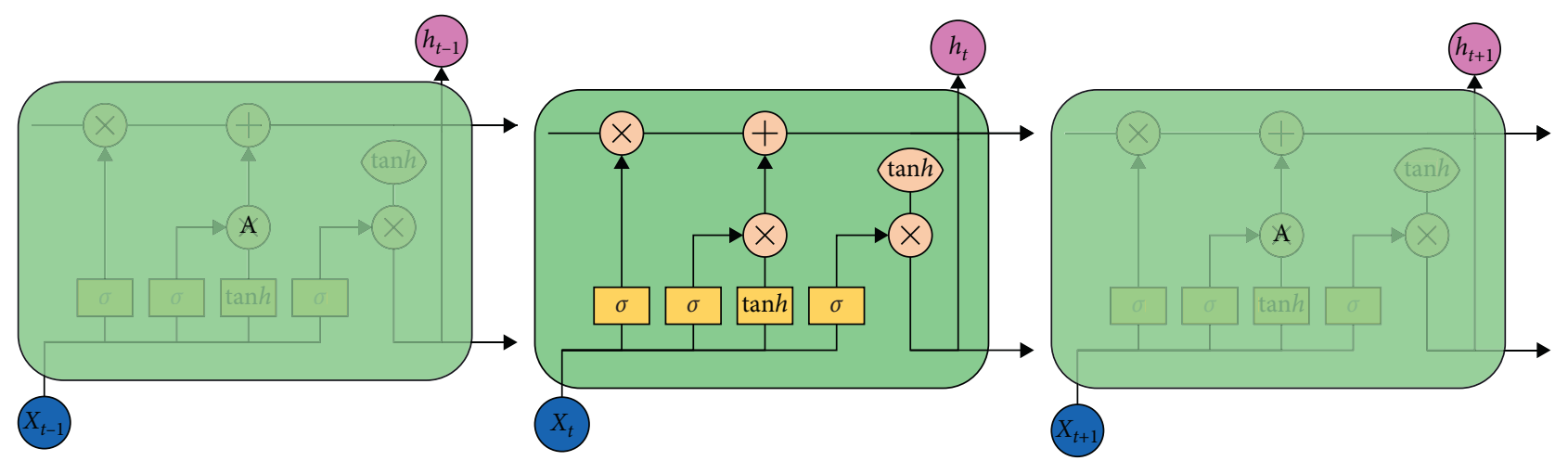

FIGURE 4: The structure of the long short-term memory (LSTM).

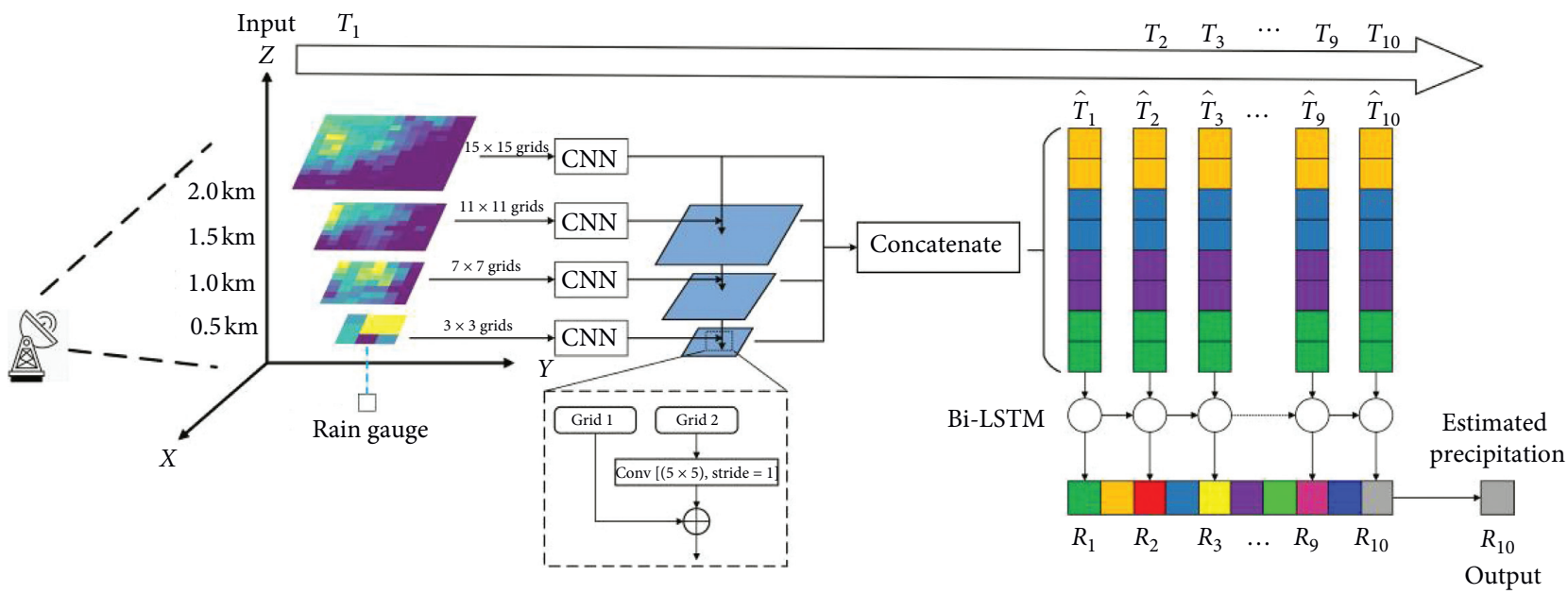

FIGURE 5: The framework of the spatiotemporal deep fusion model.

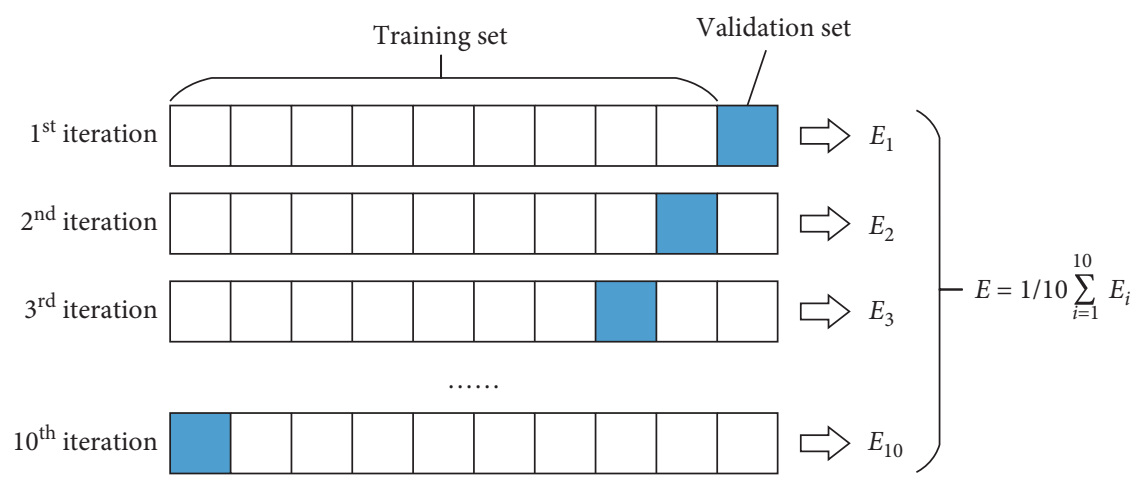

FIGURE 6: Schematic diagram of ten-fold cross-validation.

$$
\begin{aligned}
\text { Loss }_{\mathrm{MSE}} & =\frac{1}{m} \sum_{i=1}^{m}\left(P_{\mathrm{obs}}^{i}-P_{\mathrm{pre}}^{i}\right)^{2}, \\
\text { Loss } \__{10} & =\frac{1}{m_{T_{10}}} \sum_{i=1}^{m_{T_{10}}}\left(P_{\mathrm{obs}}^{i}-P_{\mathrm{pre}}^{i}\right),
\end{aligned}
$$

Loss_Sum $=$ Loss $\__{-} T_{1}+$ Loss_ $T_{2}+\cdots+$ Loss $_{-} T_{10}$.

Loss_ $T_{10}$ is the error between the estimated value and the true value in the last time point. Loss_Sum is the sum of the errors in 10 time stamps. In addition, the learning rate update strategy was introduced in the model training stage, and the learning rate was updated every 10 iterations. The error in the training phase was trained using the gradient descent method, and the parameters of the model were updated to minimize the error of each epoch. Various hyperparameter combinations were tested, the learnable parameters of the model were optimized, and the lowest MSE was obtained. Finally, a minibatch size of 20 and an epoch size of 150 were determined through the minimizing processes. 
2.4. Evaluation Parameters. The mean value of the 10 trained models was used to evaluate the performance of each rain gauge. The root mean square error (RMSE), mean bias (MB), mean absolute error (MAE), and correlation coefficient (CC) were selected as the 6-minute time-scale precipitation estimation criteria. The specific calculation formulas are as follows:

$$
\begin{aligned}
\mathrm{RMSE} & =\sqrt{\frac{\sum_{i=1}^{n}\left(P_{\mathrm{pre}}^{i}-P_{\mathrm{obs}}^{i}\right)^{2}}{n}}, \\
\mathrm{MB} & =\frac{\sum_{i=1}^{n}\left(P_{\mathrm{pre}}^{i}-P_{\mathrm{obs}}^{i}\right)}{n}, \\
\mathrm{MAE} & =\frac{\sum_{i=1}^{n}\left|P_{\mathrm{pre}}^{i}-P_{\mathrm{obs}}^{i}\right|}{n}, \\
\mathrm{CC} & =\frac{\sum_{i=1}^{n}\left(P_{\mathrm{pre}}^{i}-P_{\mathrm{pre}}^{-}\right)\left(P_{\mathrm{obs}}^{i}-P_{\mathrm{obs}}^{-}\right)}{\sqrt{\sum_{i=1}^{n}\left(P_{\mathrm{pre}}^{i}-P_{\mathrm{pre}}^{-}\right)^{2} \sum_{i=1}^{n}\left(P_{\mathrm{obs}}^{i}-P_{\mathrm{obs}}^{-}\right)^{2}}} .
\end{aligned}
$$

In equations (12)-(15), $P_{\mathrm{obs}}^{i}$ is the measured value of the rain gauge $(\mathrm{mm} / 6 \mathrm{~min}), P_{\text {pre }}^{i}$ is the estimated value of the model $(\mathrm{mm} / 6 \mathrm{~min}), P_{\mathrm{obs}}^{-}$and $P_{\text {pre }}^{-}$are the mean values of $P_{\text {obs }}^{i}$ and $P_{\text {pre }}^{i}(\mathrm{~mm} / 6 \mathrm{~min})$, respectively, and $\mathrm{n}$ is the number of all observations.

The threat score (TS) was selected as the criterion for evaluating the quality of the precipitation estimation. According to the classification standard proposed by Zhang et al. [66], the 6-minute time scale of the precipitation was divided into 5 different grades (Table 2), allowing the TS for precipitation of all intensities to be determined. The TSs are

$$
T S_{k}=\frac{N A_{k}}{N A_{k}+N B_{k}+N C_{k}} \times 100 \%, \quad \text { (range : from } 0 \text { to } 1 \text {; desirable value } 1 \text { ), }
$$

where the $k$ values range from 1 to 4 , meaning that the rainfall estimated by the model is $\geq 0.1 \mathrm{~mm}, \geq 0.7 \mathrm{~mm}$, $\geq 1.5 \mathrm{~mm}$, and $\geq 4 \mathrm{~mm}$, respectively. $\mathrm{NA}, \mathrm{NB}$, and $\mathrm{NC}$ are the hit counts (true positive events), false alarms (false positive events), and miss times (missing events), respectively.

\section{Results and Discussion}

3.1. Performances of Different Models for Precipitation Estimation. To illustrate the priority of the proposed model, the Ordinary Kriging (OK) interpolation based on Spherical variogram [19], $Z=300 R^{1.4}(Z-\mathrm{R}(1)), Z-R$ relationship based on the optimization algorithm $(Z-R(2))$ [35], the MLP model, the FFNet model, and the LSTM model were selected for comparison with the proposed model. The CAPPI data at an elevation of $2.0 \mathrm{~km}$ were used as the input of the FFNet (FFNet (Grid $2.0 \mathrm{~km}$ )) to verify the effectiveness of the FFNet model's feature fusion. Two loss functions (Loss_ $T_{10}$ and Loss_Sum) were considered in the model with the LSTM unit.

As can be seen from Figure 7 and Table 3, the correlation of the $\mathrm{OK}$ interpolation based on the cross-validation method [67] is the lowest, the TSs of the four precipitation categories are the lowest, and the TSs of the rainstorm and downpour are less than 5\%. The degree of precipitation's stationarity decreases as the amount of precipitation increases, and the increase in precipitation sometimes leads to a decrease in the correlation described by the semivariogram, which affects the quality of the precipitation interpolation [18]. The RMSE, MAE, and MB of the $Z-R$ (1) are the largest, indicating that the estimated rainfall of the $Z-R$ (1) is higher than the rain gauge value, and the error is the largest. Therefore, the fixed $Z-R$ relationship does not apply to all regions of China. The $Z-R$ (2) based on the optimization algorithm is more suitable for the study area than the fixed-relationship $Z-R(1)$, and its error is less than that of the $Z-R$ (1). In addition, the quality of the precipitation estimate of the $Z-R$ (2) is better than that of the $Z-R$ (1), and in particular, the estimate for the downpour increased by $87 \%$. The TSs of the two $Z-R$ relationships for light/moderate rain, heavy rain, and rainstorms are all greater than $50 \%$, which is one of the reasons that the $Z-R$ relationship is an effective algorithm for radar QPE, which is consistent with the results of other researchers [45]. The error of the MLP model is higher than that of all the other deep learning networks, and the TSs of the 4 categories of rainfall are only higher than those of the two $Z-R$ relationships. This may be because the MLP only fits the radar data and precipitation data nonlinearly, and it does not consider their temporal and spatial correlations [68]. The error of the FFNet model is lower than that of the MLP, indicating that the spatial information has a significant improvement effect on the radar QPE. The RMSE and MAE of the FFNet (Grid $2.0 \mathrm{~km}$ ) are significantly higher than those of the FFNet, and its TSs for heavy rain and rainstorms are the lowest among all of the models, which is due to the low correlation between the high elevation radar reflectivity information and the surface precipitation [69] and the fact that it only uses a single layer of reflectivity data and ignores the spatial structure of the rainfall system and the effectiveness of the rainfall feature fusion. When the LSTM (LSTM (Loss_T 10 ) and LSTM (Loss_Sum)) models are used to estimate the precipitation, only the time dependence of the precipitation is considered, and the error is lower than that of the MLP and $Z-R$ relationships. Therefore, the temporal correlation of the precipitation can also improve the accuracy of the radar QPE. Moreover, the error of the LSTM model using the loss function Loss_Sum is less than that using the Loss_ $T_{10}$, which is because the sum of the loss values of multiple timestamps can reflect the overall performance of the fusion precipitation within one hour, so the network can better learn the correlation between the time series data, correct the precipitation estimation errors of the past timestamps in advance, and improve the overall accuracy of the estimate of the fusion precipitation intensity. Therefore, the MB of the LSTM (Loss_Sum) is the smallest among all the models. The proposed model using the loss function Loss_Sum (FFNet-LSTM (Loss_Sum)) fully considers the dependence of the precipitation on both time and space, and it is better than all of the comparable models. The corresponding RMSEs and MAEs are the lowest, i.e., $2.52 \mathrm{~mm} / 6 \mathrm{~min}$ and $0.66 \mathrm{~mm} / 6 \mathrm{~min}$ lower than the $Z-R(1)$, 
TABLE 2: Categories of 6-minute rainfall.

\begin{tabular}{lccccc}
\hline Category & Drizzle & Light/moderate rain & Heavy rain & Rainstorm & Downpour \\
\hline 6-minute rainfall $(\mathrm{mm})$ & {$[0,0.1)$} & {$[0.1,0.7)$} & {$[0.7,1.5)$} & {$[1.5,4)$} & {$[4,+\infty)$} \\
\hline
\end{tabular}

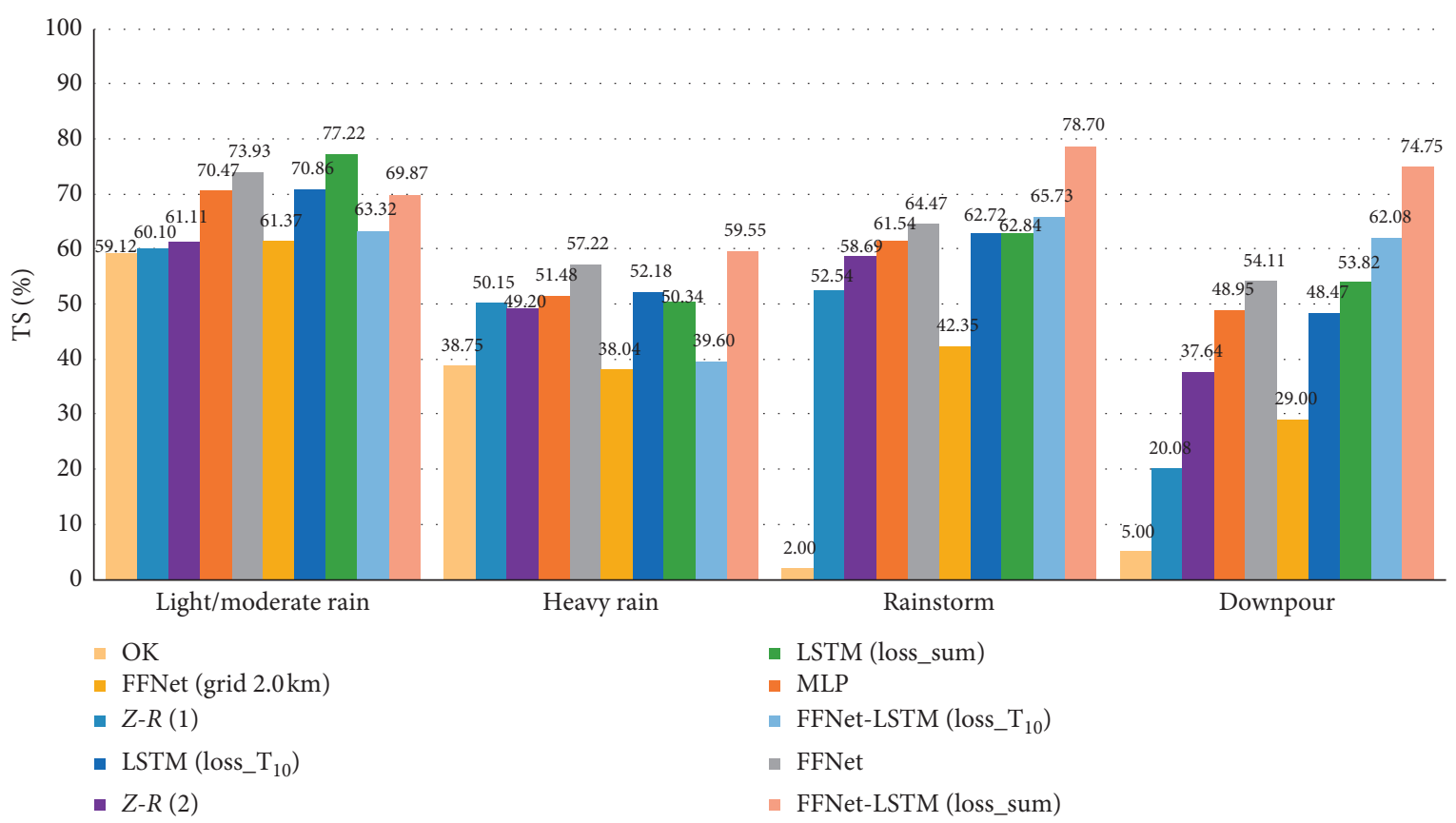

FIGURE 7: TSs of the precipitation estimates for four categories of rainfall obtained using different models.

TABLE 3: Evaluation results of the different models.

\begin{tabular}{lcccc}
\hline & RMSE $(\mathrm{mm} / 6 \mathrm{~min})$ & CC & MAE $(\mathrm{mm} / 6 \mathrm{~min})$ & MB $(\mathrm{mm} / 6 \mathrm{~min})$ \\
\hline OK & 2.90 & 0.31 & 0.52 & 0.24 \\
$Z-R(1)$ & 2.96 & 0.65 & 0.84 & 0.55 \\
Z-R $(2)$ & 0.95 & 0.70 & 0.44 & 0.13 \\
MLP & 0.72 & 0.74 & 0.35 & -0.05 \\
FFNet & 0.70 & 0.76 & 0.28 & 0.03 \\
FFNet (Grid 2.0 km) & 0.84 & 0.60 & 0.36 & 0.04 \\
LSTM (Loss_T10) & 0.71 & 0.75 & 0.25 & -0.01 \\
LSTM (Loss_Sum) & 0.68 & 0.77 & 0.24 & $\mathbf{0 . 0 0}$ \\
FFNet-LSTM (Loss_T10) & 0.65 & 0.79 & 0.21 & -0.03 \\
FFNet-LSTM (Los__Sum) & $\mathbf{0 . 4 4}$ & $\mathbf{0 . 8 5}$ & $\mathbf{0 . 1 8}$ & -0.01 \\
\hline
\end{tabular}

Bold font represents the best performance.

respectively; and the correlation coefficient increases from 0.65 to 0.85 . The TSs of the FFNet-LSTM (Loss_Sum) model for heavy rain, rainstorms, and downpours are the highest among all of the models.

3.2. Spatial Distribution of the Metrics for the Different Models. The $Z-R(1)$ and MLP are the most representative radar QPE models in terms of meteorology and deep learning, respectively. Therefore, they are compared with the FFNetLSTM (Loss_Sum) to assess the spatial distribution of the metrics. The spatial distributions of the metrics for the three models' 6-minute precipitation estimations over the study area are shown in Figure 7. Based on the observations from the 260 Automatic Weather Stations (AWSs), the CC,
RMSE, MAE, and MB of the precipitation estimates obtained using the Z-R (1), MLP model, and FFNet-LSTM (Loss_Sum) were calculated for each gauge, and the gauge values were interpolated into others using the inverse distance weighting (IDW) interpolation to obtain the spatial distribution of the metrics [70]. As shown in Figure 8, the correlation coefficients of the Z-R (1) and MLP model have a large area of low values in the center of the study area, while the FFNet-LSTM (Loss_Sum) reduces the area of the low CC values in the center area and further improves the overall correlation coefficient of the study area. The MAE, RMSE, and $\mathrm{MB}$ values of the $Z-R(1)$ are the highest among all of the models in the study area, indicating that there is a large discrepancy between the estimated values and the reference values and that the amount of precipitation is overestimated. 



Figure 8: Spatial distributions of the metrics of the Z-R (1), MLP, and FFNet-LSTM (Loss_Sum) over the Shijiazhuang: (a) correlation coefficient (CC), (d) root mean square error (RMSE), (g) mean absolute error (MAE), and (j) Mean bias (MB) for the $Z$ - $R$ (1); (b) CC, (e) RMSE, (h) MAE, and (k) MB for the MLP; and (c) CC, (f) RMSE, (i) MAE, and (l) MB for the FFNet-LSTM (Loss_Sum).

The spatial distributions of the RMSE, MAE, and MB values of the MLP and FFNet_LSTM (Loss_Sum) are similar, but the error value of the FFNet-LSTM (Loss_Sum) is lower than that of the MLP overall. The three models all have different degrees of unevenness in terms of the distributions of their metric values. This is due to two possible factors. First, the distribution of the AWSs is uneven, and the actual precipitation measurement error cannot be used in areas with sparse stations. Second, the simple quality control algorithms have limitations in solving the problem of the AWS data quality improvement [71].
3.3. Performance of the Models on Different Time Scales. Figure 9 shows the detection and estimation of the rainfall intensity in different evolution stages of a rainfall event that occurred in Shijiazhuang from 8:00 UTC on June 8th to 16: 00 UTC on June 9th in 2018 obtained using the proposed model and the comparison models. Time-series plots for the hourly rainfall estimates obtained using the AWSs, $Z-R(1)$, MLP, FFNet, LSTM (Loss_Sum), and FFNet-LSTM (Loss_Sum) are shown in Figure 9(a). The $Z-R$ (1) almost overestimates the entire rainfall event. The MLP underestimates the rainfall intensity during multiple periods of the 


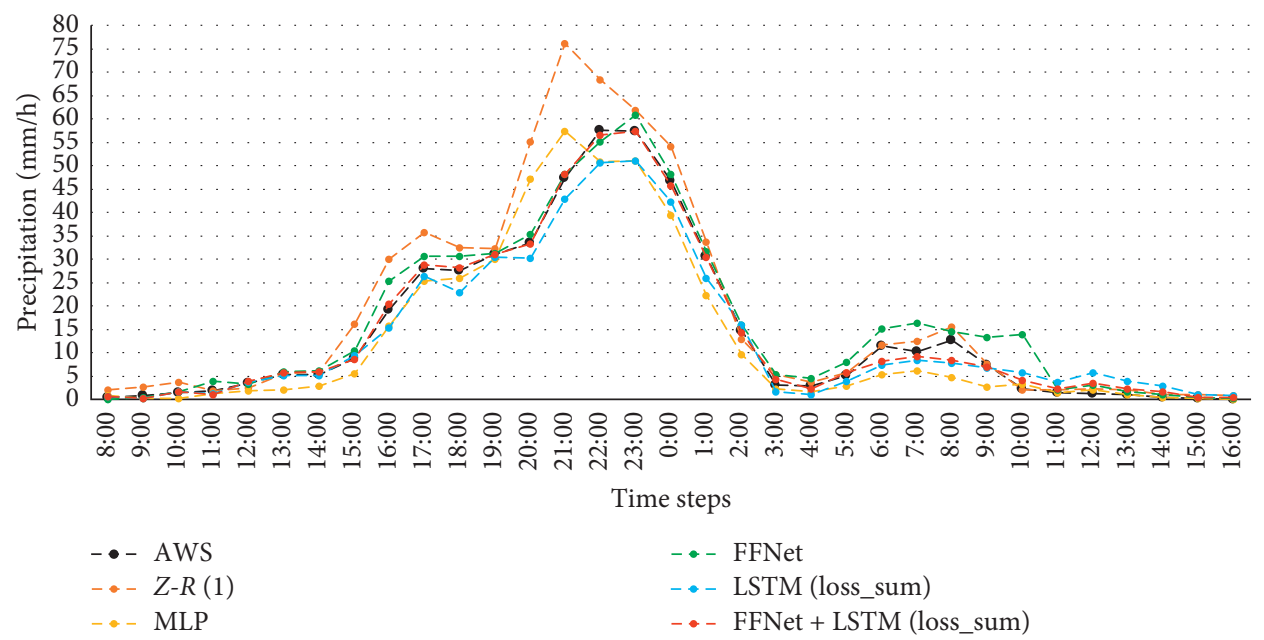

(a)

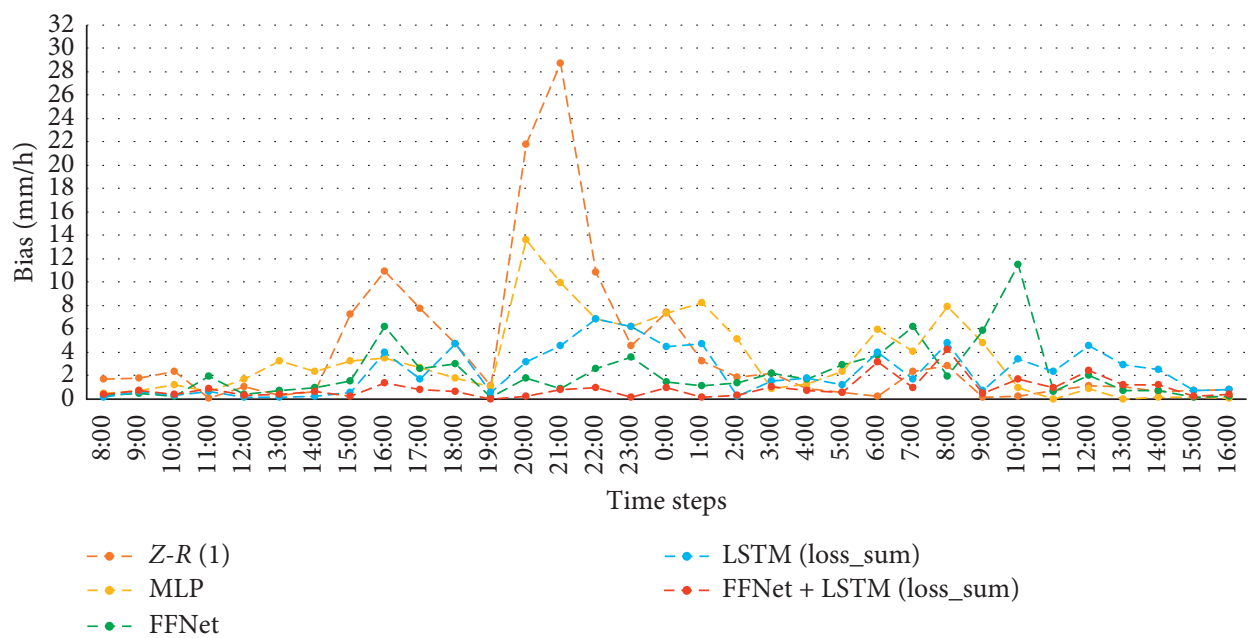

(b)

Figure 9: Time series plots of (a) the hourly rainfall estimates and (b) the positive bias ( $\mathrm{mm} / \mathrm{h}$ ) derived from the AWSs for the Z-R(1), MLP, FFNet, LSTM (Loss_Sum), and FFNet-LSTM (Loss_Sum) models throughout the evolution of the precipitation event from 0800 UTC on 8 June to 1600 on 9 June 2018.

rainfall process. The FFNet improves the accuracy of the precipitation estimation, but the results are not good during the period when the rainfall intensity changes suddenly. The LSTM (Loss_Sum) underestimates the rainfall intensity during the most intense period of rainfall. The estimate of the FFNet-LSTM (Loss_Sum) is in good agreement with the AWS observations although there was a slight underestimation from 0500 to 0900 UTC on June 9. During periods of heavy precipitation, the positive deviation of the model tends to increase, but the trend of the FFNet-LSTM (Loss_Sum) is the least obvious (Figure 9(b)). The Z-R (1), MLP, FFNet, and LSTM (Loss_Sum) models have maximum positive bias values of $28.7,13.6,11.5$, and $6.9 \mathrm{~mm} / \mathrm{h}$, respectively. However, the positive bias of the FFNet-LSTM (Loss_Sum) is significantly smaller than that of the comparison models (about $5 \mathrm{~mm} / \mathrm{h}$ ).

Quantitative precipitation estimates on a longer time scale are of great significance for studying water resource changes during the flood season and for coping with secondary disasters caused by precipitation $[50,51,72]$. Figure 10 demonstrates how the proposed model and the comparison models perform in terms of detecting and estimating the daily rainfall intensity in Shijiazhuang during the flood season from July 7 to September 1, 2018. Similar to the quantitative hourly precipitation estimation, the $Z-R(1)$ overestimates the amount of rainfall during the flood season. The precipitation estimated by the MLP is lower than the measured values from the AWSs. The evaluation results of the FFNet and LSTM (Loss_Sum) are similar, and underestimation occurs during periods of high rainfall intensity. The estimated values of the FFNet-LSTM (Loss_Sum) correspond well with the AWS observations although there is a slight underestimation when there is a sudden change in the rainfall intensity (Figure 10(a)). The maximum values of the positive bias for the Z-R (1), MLP, FFNet, LSTM (Loss_Sum), and FFNet-LSTM (Loss_Sum) models are about 108.1, 121.1, 114.2, 66.6, and $34.6 \mathrm{~mm} / \mathrm{d}$, respectively; and the numbers of days when the positive deviation is less 


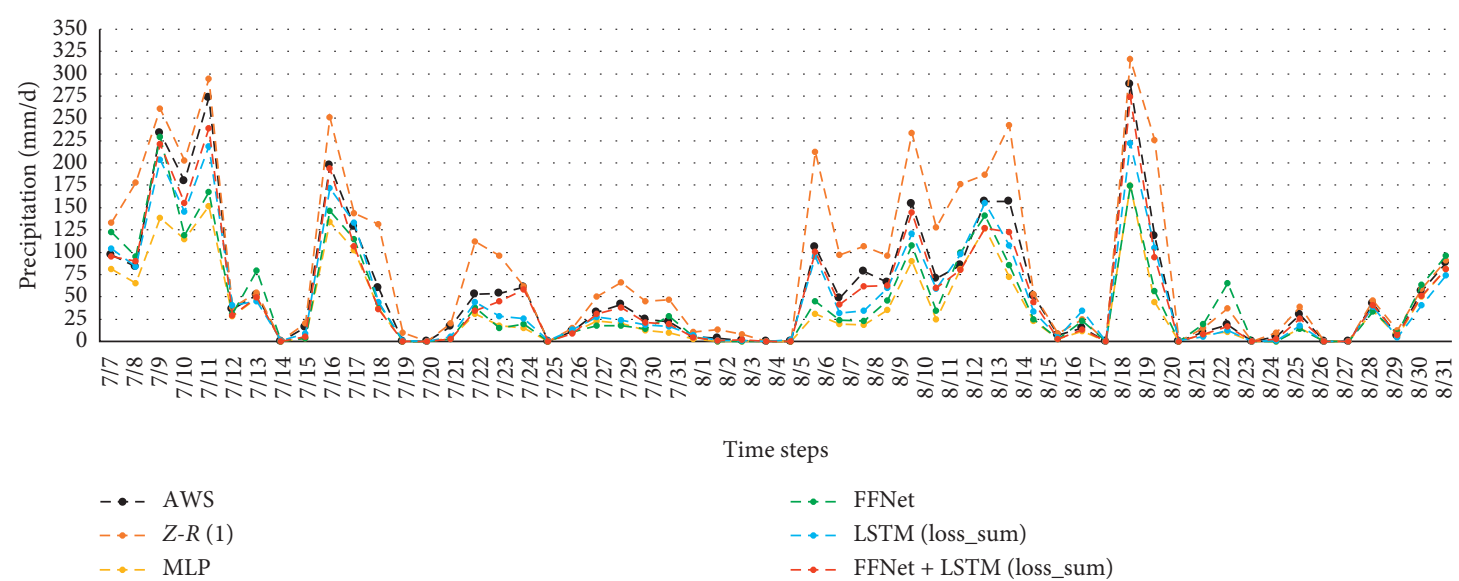

(a)

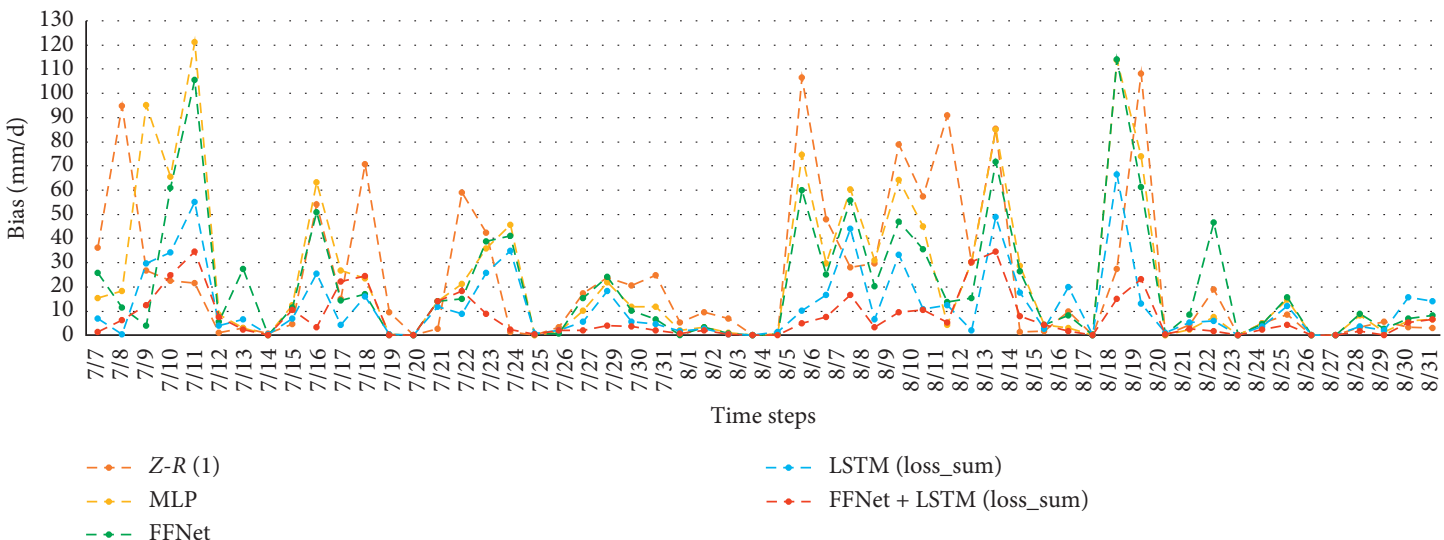

(b)

Figure 10: Time series plots of (a) the daily rainfall estimates and (b) the positive bias ( $\mathrm{mm} / \mathrm{d}$ ) derived from the AWSs for the $Z$ - $R$ (1), MLP, FFNet, LSTM (Loss_Sum), and FFNet-LSTM (Loss_Sum) models throughout the evolution of the precipitation event from UTC 7 July to 1 Sep 2018.

than $5 \mathrm{~mm} / \mathrm{d}$ are $19,21,22,24$, and 33 , respectively (Figure 10(b)). The estimated precipitation time series of the model on the hourly and daily time scales show that the proposed model can estimate long-term precipitation events very well, and it can accurately describe the rainfall in the flood season.

\section{Conclusions}

Considering the spatial correlation and time dependence of precipitation and recognizing the advantages of the FFNet in feature fusion and extraction and those of the LSTM in capturing the time dependence, an offline radar QPE method based on the FFNet and LSTM was developed. First, considering the temporal and spatial characteristics of precipitation, radar CAPPI data were selected and threedimensional radar data were used as the input for the feature fusion and extraction. Second, the dependence of the time series radar data was captured based on the sensitivity of the LSTM to the sequence data. Finally, the FFNet and LSTM were creatively combined, and it was found that the loss sum based on the time series data (Loss_Sum) greatly improves the accuracy of the precipitation estimation. The experimental results show that the proposed FFNet-LSTM method is significantly better than the basic estimation methods.

In addition, the FFNet-LSTM provides a new perspective for the utilization of high-elevation radar data and quantitative precipitation estimation in an accurate and effective way. Based on the better performance of the FFNet-LSTM model with regard to the spatial distributions of the metrics and precipitation estimations on different time scales, we conclude that once it is trained and large-scale data are input, it can be applied to the radar QPE of a large area and a long time series. In future investigations, it will be interesting to investigate how the FFNet-LSTM model performs in farfrom-sample situations associated with climate variability and changes. If successful, it may provide a new technical choice for quantitative precipitation estimation based on the Doppler radar.

\section{Data Availability}

The Doppler weather radar data, national weather stations (NWSs) data, and automatic weather stations (AWSs) data used to support the findings of this study were supplied by 
Shijiazhuang Meteorological Observatory under license and so cannot be made freely available.

\section{Conflicts of Interest}

The authors declare that there are no conflicts of interest regarding the publication of this paper.

\section{Acknowledgments}

This study was supported by the National Natural Science Foundation of China (Grant nos. 41875027 and 41871238), National Key Research and Development Program of China (Grant no. 2018YFC1506502), Jiangsu Postgraduate Practice Innovation Program (Grant no. SJCX21_0367), and Shijiazhuang Meteorological Bureau (Grant no. SJZQXJHT2019-45).

\section{References}

[1] Z. Jian, Y. Qi, C. Langston et al., "A real-time algorithm for merging radar QPEs with rain gauge observations and orographic precipitation climatology," Journal of Hydrometeorology, vol. 15, no. 5, pp. 1794-1809, 2014.

[2] S. J. Cole and R. J. Moore, "Hydrological modelling using rain gauge and radar-based estimators of areal rainfall," Journal of Hydrology, vol. 358, no. 3-4, pp. 159-181, 2008.

[3] T. H. Syed, V. Lakshmi, E. Paleologos et al., "Analysis of process controls in land surface hydrological cycle over the continental United States," Journal of Geophysical Research: Atmospheres, vol. 109, no. D22, pp. 1-11, 2004.

[4] A. S. Gebregiorgis and F. Hossain, "Understanding the dependence of satellite rainfall uncertainty on topography and climate for hydrologic model simulation," IEEE Transactions on Geoscience and Remote Sensing, vol. 51, no. 1, pp. 704-718, 2013.

[5] Y. Tang, X. Yang, W. Zhang, and G. Zhang, "Radar and rain gauge merging-based precipitation estimation via geographical-temporal attention continuous conditional random field," IEEE Transactions on Geoscience and Remote Sensing, vol. 56, no. 9, pp. 5558-5571, 2018.

[6] G. Delrieu, I. Braud, A. Berne et al., "Weather radar and hydrology," Advances in Water Resources, vol. 32, no. 7, pp. 969-974, 2009.

[7] J. Simpson, R. F. Adler, and G. R. North, "A proposed tropical rainfall measuring mission (TRMM) satellite," Bulletin of the American Meteorological Society, vol. 69, no. 3, pp. 278-295, 1988.

[8] R. J. Joyce, J. E. Janowiak, P. A. Arkin, and P. Xie, “CMORPH: A method that produces global precipitation estimates from passive microwave and infrared data at high spatial and temporal resolution," Journal of Hydrometeorology, vol. 5, no. 3, pp. 487-503, 2004.

[9] Y. Wang and V. Chandrasekar, "Quantitative precipitation estimation in the CASA X-band dual-polarization radar network," Journal of Atmospheric and Oceanic Technology, vol. 27, no. 10, pp. 1665-1676, 2010.

[10] R. A. Scofield and R. J. Kuligowski, "Status and outlook of operational satellite precipitation algorithms for extremeprecipitation events," Weather and Forecasting, vol. 18, no. 6, pp. 1037-1051, 2003.

[11] M. Steiner, J. A. Smith, S. J. Burges, C. V. Alonso, and R. W. Darden, "Effect of bias adjustment and rain gauge data quality control on radar rainfall estimation," Water Resources Research, vol. 35, no. 8, pp. 2487-2503, 1999.

[12] S.-S. Yoon, A. T. Phuong, and D.-H. Bae, "Quantitative comparison of the spatial distribution of radar and gauge rainfall data," Journal of Hydrometeorology, vol. 13, no. 6, pp. 1939-1953, 2012.

[13] Z. W. Wang, H. Chen, S. Yuan et al., "A new generation of refined detection technology for dual polarization weather mine (CINRAD/SAD)," Meteorological Science and Technology, vol. 48, no. 03, pp. 331-336, 2020.

[14] J. Li and A. D. Heap, "A review of spatial interpolation methods for environmental scientists," Australian Geological Survey Organisation, vol. 68, p. 154, 2008.

[15] A. Bárdossy and J. Li, "Geostatistical interpolation using copulas," Water Resour Research, vol. 44, no. 7, p. W07412, 2008.

[16] C. Rusu and V. Rusu, "Radial basis functions versus geostatistics in spatial interpolations," IFIP International Conference on Artificial Intelligence in Theory and Practice, vol. 217, pp. 119-128, 2006.

[17] G. Y. Lu and D. W. Wong, "An adaptive inverse-distance weighting spatial interpolation technique," Computers \& Geosciences, vol. 34, no. 9, pp. 1044-1055, 2008.

[18] D. Chen, T. Ou, L. Gong et al., "Spatial interpolation of daily precipitation in China: 1951-2005," Advances in Atmospheric Sciences, vol. 27, no. 6, pp. 1221-1232, 2010.

[19] Q. Wang, C. Xu, and H. Chen, "Comparison and analysis of different variogram functions models in kriging interpolation of daily rainfall," Journal of Water Resources Research, vol. 5, no. 5, pp. 469-477, 2016.

[20] C. Kidd, A. Becker, G. J. Huffman et al., "So, how much of the earth's surface is covered by rain gauges?" Bulletin of the American Meteorological Society, vol. 98, no. 1, pp. 69-78, 2017.

[21] S. Rana, J. Mcgregor, and J. Renwick, "Precipitation seasonality over the Indian subcontinent: an evaluation of gauge, reanalyses, and satellite retrievals," Journal of Hydrometeorology, vol. 16, no. 2, pp. 631-651, 2015.

[22] D. P. Jorgensen and P. T. Willis, "A Z-R relationship for hurricanes," Journal of Applied Meteorology, vol. 21, no. 3, pp. 356-366, 1982.

[23] J. S. Marshall and W. M. K. Palmer, "The distribution of raindrops with size," Journal of Meteorology, vol. 5, no. 4, pp. 165-166, 1948.

[24] J. S. Marshall, R. C. Langille, and W. M. K. Palmer, "Measurement of rainfall by radar," Journal of Meteorology, vol. 4, no. 6, pp. 186-192, 1947.

[25] D. Rosenfeld, D. B. Wolff, and D. Atlas, "General probabilitymatched relations between radar reflectivity and rain rate," Journal of Applied Meteorology, vol. 32, no. 1, pp. 50-72, 1993.

[26] B. E. Vieux and P. B. Bedient, "Estimation of rainfall for flood prediction from WSR-88D reflectivity: a case study, 17-18 october 1994*," Weather and Forecasting, vol. 13, no. 2, pp. 407-415, 1998.

[27] J. W. Ryde, "The attenuation of centimetre radio waves and the echo intensities resulting from atmospheric phenomena," Journal of the Institution of Electrical Engineers-Part IIIA: Radiolocation, vol. 93, no. 1, pp. 101-103, 1946.

[28] W. L. Crosson, C. E. Duchon, R. Raghavan, and S. J. Goodman, "Assessment of rainfall estimates using a Standard $Z-R$ relationship and the probability matching method applied to composite radar data in central Florida," Journal of Applied Meteorology, vol. 35, no. 8, pp. 1203-1219, 1996. 
[29] A. S. Chumchean and A. Sharma, "Correcting of real-time radar rainfall bias using a Kalman filtering approach," Journal of Hydrology, vol. 317, no. 1-2, pp. 123-137, 2006.

[30] D. Atlas, C. W. Ulbrich, F. D. MarksJr., E. Amitai, and C. R. Williams, "Systematic variation of drop size and radarrainfall relations," Journal of Geophysical Research: Atmospheres, vol. 104, no. D6, pp. 6155-6169, 1999.

[31] G. W. Lee and I. Zawadzki, "Variability of drop size distributions: time-scale dependence of the variability and its effects on rain estimation," Journal of Applied Meteorology and Climatology, vol. 44, no. 2, pp. 241-255, 2005.

[32] O. P. Prat and A. P. Barros, "Exploring the transient behavior of $Z-R$ relationships: implications for radar rainfall estimation," Journal of Applied Meteorology and Climatology, vol. 48, no. 10, pp. 2127-2143, 2009.

[33] C. W. Ulbrich and L. G. Lee, "Rainfall measurement error by WSR-88D radars due to variations inZ-RLaw parameters and the radar constant," Journal of Atmospheric and Oceanic Technology, vol. 16, no. 8, pp. 1017-1024, 1999.

[34] E. A. Brandes and A. Edward, "Optimizing rainfall estimates with the aid of radar," Journal of Applied Meteorology, vol. 14, no. 7, pp. 1339-1345, 1975.

[35] X. Q. Yuan, G. H. Ni, A. J. Pan et al., "Research on the Z-R relationship of Beijing's new generation weather radar based on optimization algorithm," Hydrology, vol. 30, no. 01, pp. 1-6, 2010, in Chinese.

[36] S. Ramli, S. H. Abu Bakar, and W. Tahir, "Radar hydrology: new Z/R relationships for Klang River Basin, Malaysia based on rainfall classification," in Proceedings of the 2011 IEEE Colloquium on Humanities, Science and Engineering, pp. 537-541, Penang, Malaysia, December 2011.

[37] S. Chumchean, A. Sharma, and A. Seed, "An integrated approach to error correction for real-time radar-rainfall estimation," Journal of Atmospheric and Oceanic Technology, vol. 23, no. 1, pp. 67-79, 2006.

[38] S. Thorndahl, J. E. Nielsen, and M. R. Rasmussen, "Bias adjustment and advection interpolation of long-term high resolution radar rainfall series," Journal of Hydrology, vol. 508, pp. 214-226, 2014.

[39] Y. Gou, Y. Ma, H. Chen, and Y. Wen, "Radar-derived quantitative precipitation estimation in complex terrain over the eastern Tibetan Plateau," Atmospheric Research, vol. 203, pp. 286-297, 2018.

[40] V. Chandrasekar, K. S. Ramanujam, H. Chen et al., "Rainfall estimation from spaceborne and ground based radars using neural networks," in Proceedings of the 2014 IEEE Geoscience and Remote Sensing Symposium, pp. 4966-4969, Quebec City, Canada, July 2014.

[41] A. Kusiak, X. Wei, A. P. Verma, and E. Roz, "Modeling and prediction of rainfall using radar reflectivity data: a datamining approach," IEEE Transactions on Geoscience and Remote Sensing, vol. 51, no. 4, pp. 2337-2342, 2013.

[42] H. Ayat, M. R. Kavianpour, S. Moazami et al., "Calibration of weather radar using region probability matching method (RPMM)," Theoretical and Applied Climatology, vol. 134, no. 1, pp. 165-176, 2018.

[43] B.-J. Huang, T.-H. Tseng, and C.-M. Tsai, "Rainfall estimation in weather radar using support vector machine," Intelligent Information and Database Systems, vol. 9011, pp. 583-592, 2015.

[44] J. Li, A. D. Heap, A. Potter, and J. J. Daniell, “Application of machine learning methods to spatial interpolation of environmental variables," Environmental Modelling \& Software, vol. 26, no. 12, pp. 1647-1659, 2011.
[45] H. Liu, V. Chandrasekar, G. Xu et al., "An adaptive neural network scheme for radar rainfall estimation from WSR-88D observations," Journal of Applied Meteorology, vol. 40, no. 11, pp. 2038-2050, 2001.

[46] W. Li, V. Chandrasekar, and G. Xu, "Investigations in radar rainfall estimation using neural networks," IEEE International Geoscience and Remote Sensing Symposium, vol. 4, pp. 2347-2349, 2003.

[47] R. Xiao and V. Chandrasekar, "Development of a neural network based algorithm for rainfall estimation from radar observations," IEEE Transactions on Geoscience and Remote Sensing, vol. 35, no. 1, pp. 160-171, 1997.

[48] T. B. Trafalis, M. B. Richman, A. White, and B. Santosa, "Data mining techniques for improved WSR-88D rainfall estimation," Computers \& Industrial Engineering, vol. 43, no. 4, pp. 775-786, 2002.

[49] Y. M. Chiang, F. J. Chang, B. J. D. Jou et al., "Dynamic ANN for precipitation estimation and forecasting from radar observations," Journal of Hydrology, vol. 334, no. 1-2, pp. 250-261, 2007.

[50] E. Habib, W. F. Krajewski, and A. Kruger, "Sampling errors of tipping-bucket rain gauge measurements," Journal of $\mathrm{Hy}$ drologic Engineering, vol. 6, no. 2, pp. 159-166, 2001.

[51] H. Chen and V. Chandrasekar, "Estimation of light rainfall using Ku-band dual-polarization radar," IEEE Transactions on Geoscience and Remote Sensing, vol. 53, no. 9, pp. 5197-5208, 2015.

[52] Y. R. Shi, S. W. Shou, L. Wang et al., "Analysis of the relationship between storm relative helicity and severe convective weather types," Journal of Meteorology and Environment, vol. 27, no. 1, pp. 65-71, 2011, in Chinese.

[53] W. Q. Gao, W. C. Zhou, and Y. E. Li, "Diagnosis and analysis of wet potential vorticity in a severe convective rainstorm in North China," Journal of Meteorology and Environment, vol. 27, no. 1, pp. 1-6, 2011, in Chinese.

[54] L. R. Wang and S. S. Kuang, "Application of climatic characteristics of convective parameters in short-term forecasting," Journal of Meteorology and Environment, vol. 24, no. 5, pp. 38-41, 2008, in Chinese.

[55] Q. Zhang, Y. F. Zhao, and S. H. Fan, "Development of hourly precipitation data set for national meteorological stations in China," Heavy Rain Disaster, vol. 35, no. 2, pp. 182-186, 2016, in Chinese.

[56] L. J. Cao and Z. W. Yan, "Progresses in research of homogenization of climate data," Advances in Climate Change Research (in Chinese), vol. 7, no. 2, pp. 129-135, 2011.

[57] L. C. Montenegro, V. H. Lachos, and H. Bolfarine, "Inference for a skew extension of the Grubbs model," Statistical Papers, vol. 51, no. 3, pp. 701-715, 2010.

[58] Y. Zhang and L. J. Gao, "Underwater sensor network target tracking based on Grubbs criterion and improved particle filter algorithm," Journal of Electronics \& Information Technology, vol. 41, no. 10, pp. 2294-2301, 2019, in Chinese.

[59] Z. J. Yu and X. Zhang, "Characteristics of atmospheric water resources in Shijiazhuang area from 1970 to 2010," Journal of Hebei Normal University: Natural Science Edition, vol. 43, no. 3, pp. 256-261, 2019.

[60] L. J. Miller, C. G. Mohr, and A. J. Weinheimer, "The simple rectification to cartesian space of folded radial velocities from Doppler radar sampling," Journal of Atmospheric \& Oceanic Technology, vol. 3, no. 1, pp. 162-174, 2013.

[61] L. S. Hao, J. Z. Min, and Y. S. Shi, "Analysis of the inherent characteristics of precipitation changes in North China from 
1961 to 2008," Journal of Anhui Agricultural Sciences, vol. 38, no. 24, pp. 13269-13274+13324, 2010.

[62] Q. Cao, G. Zhang, E. Brandes, T. Schuur, A. Ryzhkov, and K. Ikeda, "Analysis of video disdrometer and polarimetric radar data to characterize rain microphysics in Oklahoma," Journal of Applied Meteorology and Climatology, vol. 47, no. 8, pp. 2238-2255, 2008.

[63] M. Donoser, M. Urschler, M. Hirzer et al., "Saliency driven total variation segmentation," in Proceedings of the 2009 IEEE 12th International Conference on Computer Vision, pp. 817824, Kyoto, Japan, October 2009.

[64] V. Mahadevan and N. Vasconcelos, "Biologically inspired object tracking using center-surround saliency mechanisms," IEEE Transactions on Pattern Analysis and Machine Intelligence, vol. 35, no. 3, pp. 541-554, 2013.

[65] S. Avidan and A. Shamir, "Seam carving for content-aware image resizing," ACM Transactions on Graphics, vol. 26, no. 3, p. 10, 2012.

[66] C. Zhang, H. Wang, J. Zeng, L. Ma, and L. Guan, “Short-term dynamic radar quantitative precipitation estimation based on wavelet transform and support vector machine," Journal of Meteorological Research, vol. 34, no. 2, pp. 413-426, 2020.

[67] R. Giraldo, P. Delicado, and J. Mateu, "Ordinary kriging for function-valued spatial data," Environmental and Ecological Statistics, vol. 18, no. 3, pp. 411-426, 2011.

[68] G. Xia and Y. Fang, "Revision modeling of precipitation estimation error for a certain type of radar based on BP artificial neural network," Ship Electronic Engineering, vol. 36, no. 11, pp. $65-67+102,2016$.

[69] H. Chen, V. Chandrasekar, H. Tan et al., "Rainfall estimation from ground radar and TRMM Precipitation Radar using hybrid deep neural networks," Geophysical Research Letters, vol. 46, no. 17-18, pp. 10669-10678, 2019.

[70] H. Wu, Q. Yang, J. Liu, and G. Wang, “A spatiotemporal deep fusion model for merging satellite and gauge precipitation in China," Journal of Hydrology, vol. 584, Article ID 124664, 2020.

[71] L. Zhu and J. Liu, "The design of quality control platform on automatic weather station," in Proceedings of 2011 International Conference on Intelligent Computing and Integrated Systems (ICISS 2011), pp. 325-328, Chongqing, China, October 2011.

[72] G. Villarini, P. V. Mandapaka, W. F. Krajewski et al., "Rainfall and sampling uncertainties: a rain gauge perspective," Journal of Geophysical Research: Atmospheres, vol. 113, no. D11, 2008. 